\section{IMPERFECT SYSTOLE AS A CAUSE OF CARDIAC DILATATION.}

Br G R A H A M S T E E L, M. D., ASSISTANT PHYSICIAN TO THE MANCHESTKR ROYAL INFLRMARY.

WHEN all the circumstances of the case are considered, it will be generally admitted that for the production of dilatation of the ventricles of the heart abnormal pressure within them during diastole is necessary. The mechanism by which this abnormal pressure is brought about is sometimes obvious -as, for instance, in aortic regurgitation. This lesion tends to the production of dilatation of the left ventricle from the outset, and hypertrophy of the walls of the ventricle is only called forth to propel the abnormal quantity of blood accommodated in the enlarged chamber. Dilatation results directly from the lesion; hypertrophy is only secondarily induced after a certain amount of dilatation has occurred. On the other hand, aortic obstruction alone has no tendency to produce dilatation of the left ventricle so long as the following two conditions are fulfilled-(1) that the ventricle remains able to complete its systole, and (2) that the micral valves remain competent. Hypertrophy of the left ventricle supplies all the compensation demanded by the circulation. Should, however, the nutrition of the heart muscle fail, either or both of these conditions will probably cease to be fulfilled, and then dilatation will occur. It has long been known as a matter of experience that incompetence of the mitral valves induces dilatation of the left ventricle-a fact which is most unequivocally observed in children who are the subjects of endocarditis. The explanation of the result is not far to seek. When there is mitral regurgitation, a certain quantity of blood is thrown back through the mitral orifice with each systole; but if the circulation is not to suffer, the same amount must be returned to the ventricle from the auricle, in addition to the normal delivery. Increased intra-ventricular pressure during diastole is thus established - dilatation is the first effect, and hypertrophy occurs secondarily.

In many cases of failing heart, in which the valves remain healthy and the muscle is the part at fault, mitral incompatence, evidenced by an apex systolic murmur, occurs early, and may thus be an important factor in the production of dilatation of the left ventricle. In other cases, however, there is not, from first to last, the slightest evidence of mitral regurgitation or of other valvular incompetence, the heart sounds remaining uniformly pure. How shall we explain the dilatation in these cases? In other words, how is the condition which we hold to be essential for the production of enlargement of the ventricle-namely, increased intraventricular pressure during diastole-established? I think we are driven to the assumption that the systole of the ventricle becomes habitually incomplete, so that the chamber is never thoroughly emptied. For this condition 1 venture, on high classical authority, to use the term "systole catalectic"-a systole stopping short ( $\kappa a \tau a \lambda \eta \kappa \tau \iota \kappa \eta$ ) of the completion of its function-in place of that commonly employed, "asystole," which would imply absence rather than incompleteness of systole. When a series of transverse sections is made through a firmly contracted left ventricle from the apex upwards, we find no evidence of a cavity until we reach a level above the musculi papillares aud open the small so-called "suprapapillary space," which always contains blood after the most perfect systole. From inattention to this space the idea has arisen that the normal ventricle by its contraction wrings out its last drop of blood, although, as we have seen, this is not absolutely the case. We have good grounds for believing that "systole catalectic" is by no means rare in the course of heart disease. In order to be convinced of this, we have only to examine the large, rounded, pouch-shaped ventricles so often encountered in the deadhouse, and try to imagine such ventricles accomplishing their systole in the normal way during the last months of life. The occurrence of "systole catalectic" seems to me to be the only explanation of dilatation of the heart without incompetence of its valves. Cases of dilated heart without valvular incompetence are, however, by no means rare. I am inclined to believe, therefore, that "systole catalectic," although al ways grave, is not only compatible with the continuance of life for prolonged periods, but that it admits of amelioration and even practical recovery.

I have at present under my care a patient the tracing of whose pulse almost demonstrates the part that "systole catalectic" may play in the production of dilated left ventricle. I subjoin the feature of this tracing which has reference to the point, and I may add that it has occurred repeatedly in various sphygmograms of the same patient in a precisely similar manner, so that there is no need of multiplying representations. The feature shown is one well known to all who are familiar with the sphygmograph. It consists of an abortive upstroke, rapid depression of the curve below the respiratory line, and, starting from the lowest level reached, an upstroke of extraoroinary height, (See sphygmogram.) The significance of these phenomena

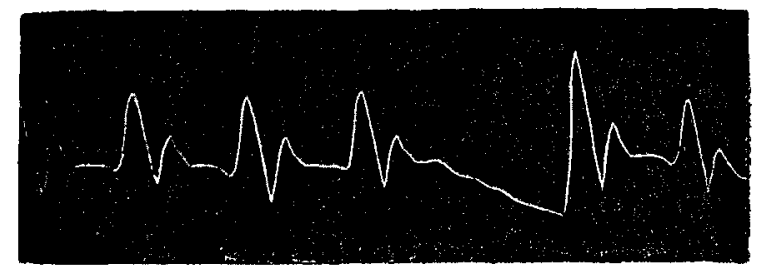

is surely as follows. An abortive very incomplete systole (often not at all represented in the radial sphygmogram) first occurs; blood accumulates rapially in the unemptied ventricle, causing its distention; but when asystole is threatening, the ventricle is roused to a supreme effort and succeeds in accomplishing its systole. The beight of the upstroke in the tracing is a measure of the abnormal amount of blood so expelled, and therefore of the distention suffered by the ventricle during its preceding diastole. Had the diastolic distention of the ventricle in this case been less acute and the ventricle less capable of $\in$ ffort, the systole might still have been effected in such a way as to admit of the continuance of life, while the diastolic increase of intra-ventricular pressure might have been little less, but the opportunity for gauging the amount of the lattex would not have been afforded us.

\section{ON A CASE OP}

\section{MENINGITIS AND THE COMPARATIVE THERAPEUTICS OF ANTIPYRIN AND ANTIFEBRIN.}

B Y ROBERT PARK, M.D., PHYSICIAN TO THE GLASGOW SAMARITAY HOSPITAL.

J. G- - aged twelve, on Nov. 29th, 1887, fell down a stair and hurt his head. There was no cut or lump raised, so much heed was not paid to it at the time, though he complained about the pain occasionally. Sixteen days afterwards he was seized with great pain in his head and vomiting, and he was unconscious and delirious for twentyfour hours. The nape of his neck was blistered, and he was freely purged. Amendment followed, so far as the more urgent symptoms were concerned. On the tenth day of his illness I was asked to see him. On Dec. 21st I found his temperature $102^{\circ}$, and his pulse 130 ; his tongue red and $d r y$; pupils equal and moderately dilated, and affected by light. He was very sensitive to light and sound. There was no strabismus, nor history of any, and no sore-throat. The stools had always been dark. There was no tympanites, the abdomen being rather retracted; and no spots. The decubitus was pleural, and he moaned frequently, and complained of the dreadful pain in the frontal and vertical part of his head. There was a history of a cardiac mitral murmur, but very careful examination failed to reveal anything of the kind now; and there was no complaint of muscular or joint pains. I diagnosed a case of localised meningitis probably due to the irritation of a clot effused at the time of the accident. Blisters were ordered behind the ears and a smart purge. The blisters were not put on, and I was asked to see the patient on the 29th. The following observations were then made:- His face is flushed; pulse 160 ; pupils moderately dilated and active; tongue red and dry. There is a history of a red rash covering the whole body, and resembling that of ecarlet fever. The mother, 